\title{
Generation of well relaxed all atom models of stereoregular polymers: a validation of hybrid particle-field molecular dynamics for polypropylene melts of different tacticities
}

\author{
Antonio De Nicola , Gianmarco Munaò , Nino Grizzuti , Finizia Auriemma , \\ Claudio De Rosa , Agur Sevink \& Giuseppe Milano
}

To cite this article: Antonio De Nicola , Gianmarco Munaò , Nino Grizzuti , Finizia Auriemma , Claudio De Rosa , Agur Sevink \& Giuseppe Milano (2020) Generation of well relaxed all atom models of stereoregular polymers: a validation of hybrid particle-field molecular dynamics for polypropylene melts of different tacticities, Soft Materials, 18:2-3, 228-241, DOI: 10.1080/1539445X.2020.1716801

To link to this article: https://doi.org/10.1080/1539445X.2020.1716801

View supplementary material ¿

Submit your article to this journal

III Article views: 128

Q View related articles $₫$

View Crossmark data $\nearrow$

Citing articles: 1 View citing articles $\widetilde{ }$ 


\title{
Generation of well relaxed all atom models of stereoregular polymers: a validation of hybrid particle-field molecular dynamics for polypropylene melts of different tacticities
}

\author{
Antonio De Nicola (i) $^{a}$, Gianmarco Munaò ${ }^{b, c}$, Nino Grizzuti ${ }^{d}$, Finizia Auriemma ${ }^{e}$, Claudio De Rosa ${ }^{e}$, Agur Sevink \\ and Giuseppe Milano ${ }^{\mathrm{a}}$
}

\begin{abstract}
aDepartment of Organic Material Science, Yamagata University, Yonezawa, Japan; 'Dipartimento di Chimica e Biologia "A. Zambelli", Leiden University, Leiden, The Netherlands

ABSTRACT

The tacticity of vinyl polymer chains strongly affects the physical properties of polymeric materials, as example the chain conformations and stiffness. In the present work we tested how the hybrid Particle-Field Molecular Dynamics (PF-MD) technique is capable to describe conformational differences of polymer chains as function of the tacticity. In particular, we focus on tacticity effect of atactic, isotactic, and syndiotactic polypropylene (PP) homopolymer melts. We found that PFMD simulations exhibit dependence of Flory's Characteristic Ratio from the fraction of racemo diads along the PP chains in qualitative agreement with Small Angle Neutron Scattering (SANS) experiments and theoretical previsions. Finally, we calculated and compared the packing length parameter on very high stereoregular syndiotactic PP systems with rheological measurements. A qualitative agreement between the calculated and experimental packing length is found.
\end{abstract} Università di Salerno, Fisciano, Italy; 'Dipartimento di Scienze Matematiche e Informatiche, Scienze Fisiche e Scienze della Terra, Università

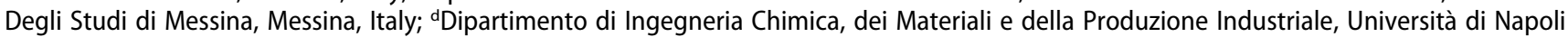

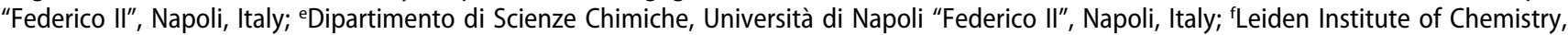

\section{ARTICLE HISTORY}

Received 29 October 2019

Accepted 12 January 2020

\section{KEYWORDS}

Tacticity; polypropylene; hybrid Particle-Field; atomistic model; molecular dynamics; packing length; Flory's characteristic ratio

\section{Introduction}

One of the crucial aspects determining the physical properties of vinyl polymers is the tacticity of the chains. A vinyl polymer chain has along its backbone a sequence of methylene $\left(\mathrm{CH}_{2}\right)$ and pseudo asymmetric methyne groups (-CHR). The stereoregularity of a vinyl polymer can be defined based on arrangement of adjacent pseudo asymmetric centers. If the two pseudo asymmetric centers, once a given direction along the polymer chain is assigned, have the same stereochemical configuration the diad (composed of two adjacent repeating units) is called meso $(\mathrm{m})$. Instead, the diad is called racemo $(r)$ in case of opposite stereochemical configuration of chiral centers (Scheme 1a). The tacticity of a vinyl polymer can be usually measured in terms of diads (or sequence of diads) distributions by using the nuclear magnetic resonance spectroscopy $\left({ }^{13} \mathrm{C}-\mathrm{NMR}\right)$ technique. ${ }^{[1-3]}$

Tuning the properties of the material, by selecting appropriate tacticity, allowed the stereoregular polypropylene (and more in general vinyl stereoregular polymers) to become one of the most relevant materials of the polymer industry. ${ }^{[4,5]}$ For semicrystalline polymeric materials, of course, tacticity is one of the key features determining most of the properties involved in several applications. Even in the case of fully amorphous materials or polymer melts, the tacticity plays an important role in influencing the final material behavior. In particular, in this case, tacticity is effective in the behavior of several properties such as: glass transition temperature $\left(T_{g}\right){ }^{[6-8]}$ self-diffusion coefficient $(D),{ }^{[8]}$ entanglement molar mass $\left(M_{e}\right),{ }^{[9]}$ and other rheological properties. ${ }^{[10,11]}$ Since tacticity mainly influences the torsional angle distributions of the polymer backbone, the main molecular effects on single chain properties are connected to changes in chain stiffness. For this reason, both experimental and theoretical studies have been conducted to understand the effect of tacticity on the stiffness of polymers. The chain stiffness can be quantified by the Flory's characteristic ratio $\left(C_{\infty}\right)$ or by the Kuhn length $(b)$. Higher values of $C_{\infty}$ correspond to stiffer chains. The stiffness of polypropylene (PP) as function of the tacticity was experimentally estimated by measuring the differences in $T_{g}{ }^{[7]}$ intrinsic viscosity in $\Theta$ solvent, ${ }^{[12,13]}$ and chain dimension by SANS measurements. ${ }^{[14,15]}$ From the theoretical point of

CONTACT Antonio De Nicola adenicola@yz.yamagata-u.ac.jp Department of Organic Material Science, Yamagata University, Yonezawa 992-8510 (-) Supplemental data for this article can be accessed here. 
(a)

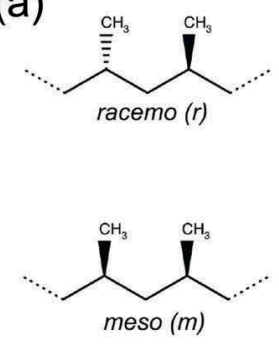

(b)
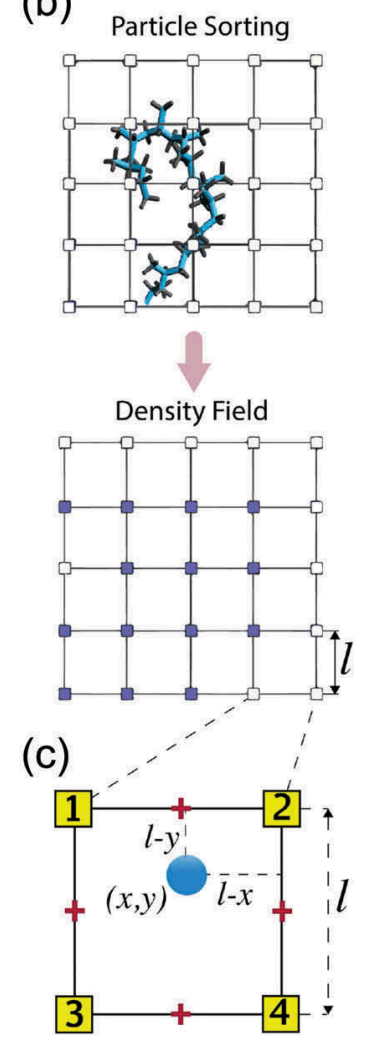

Scheme 1. (a) Chemical structure of racemo $(r)$ and meso $(m)$ diad of PP. (b) Assignment of coarse-grained density to lattice points for a PP segment's chain (for clarity only the backbone atoms have been used for the assignment). (c) Criterion for the assignment of a fraction of a particle of type $K$ to mesh vertexes numbered from 1 to 4 .

view, the Rotational Isomeric State model (RIS) applied to an unperturbed single chain was used to estimate the effect of tacticity on the PP chain dimension and conformational energy. ${ }^{[16-18]}$ Antoniadis et al. reported a Molecular Dynamics (MD) study to address the effect of tacticity on the dynamics of PP chains in melt state. ${ }^{[19,20]}$ More recently, Tzounis et al. studied the effect of tacticity on the conformational properties of PP homopolymer melts and poly(ethylene-propylene) block copolymers by using single-chain Monte Carlo (MC) technique. ${ }^{[21]}$ More generally, MD and MC techniques applied to atomistic and coarse-grained (CG) models were proposed to investigate the effect of tacticity on several physicochemical polymer properties. ${ }^{[18-26]}$ It is worth noting that one of the problems in studying polymer melts by $\mathrm{MD}$ or $\mathrm{MC}$ simulation techniques is to obtain well relaxed configurations at the equilibrium density. ${ }^{[27-29]}$ This target, due to the large relaxation times, is still out of reach of atomistic MD simulations for chain lengths typical of industrially relevant polymeric materials. Moreover, due to the large number of atom overlaps observed at the typical melt densities (which may cause divergence in the calculation of the potentials and forces), the generation of initial configurations, even for short oligomers, can be very difficult. ${ }^{[27-30]}$ Recently, some of us introduced a suitable and validated procedure based on the hybrid Particle-Field Molecular Dynamics based on a combination of MD with the Self Consistent Field theory (named in the following MD-SCF), to overcome these technical problems. ${ }^{[30]}$ In particular, this procedure was successfully applied to study different homopolymer melts and composite polymer materials at atomistic resolution and at CG level. ${ }^{[30-34]}$ However, the MD-SCF method has not been tested to study the effect of tacticity on the structural properties, such as Flory's characteristic ratio, of stereoregular homopolymer chains in melt state. This application represents a good test case for the proposed methodology, not only for the relevance of the systems, but also, from a more technical point of view, to understand if the effects of subtle differences in the chain microstructure can still be modeled using an approach based on combination of particle models with density fields.

The aim of the present work is to investigate if and how MD-SCF methods can reproduce structural properties of polymers with different tacticity and compare the results with reference atomistic MD simulations and experimental data. Such a study can provide a validation of the proposed approach and open application to further systems based on stereoregular polyolefins, which represent the largest class of industrial polymers produced by chemical industry. We have chosen PP melts as a test case because they have been deeply investigated by experiments and molecular dynamics simulations. In the following, a brief introduction to the hybrid MD-SCF method is given in the Computational Methods and Models Section. In the Results Section MD-SCF simulations are compared to reference MD and experimental data. Finally, in the Conclusion Section the main results are summarized. 


\section{Computational Methods and Models}

\section{Hybrid MD-SCF}

A brief description of the method will be given in this section, while the full description and derivation can be found in Ref. ${ }^{[30]}$ As mentioned in the Introduction, the MD-SCF approach applied in the present work has been validated and widely employed in many previous works to get equilibrated polymer melts and models of polymer composite materials on both atomistic and coarse-grained resolution. ${ }^{[2,34,35]}$ The core feature of the method is the evaluation of non-bonded forces and potentials (the most computationally expensive terms in a MD simulation) through the calculation of an external potential depending on the local density at position $V(\boldsymbol{r}) .^{[36]}$ The manybody problem in the spirit of Self Consistent Field (SCF) Theory can be reduced to a problem of deriving the partition function of a single particle in an external potential $V_{\mathrm{K}}(\boldsymbol{r}) .{ }^{[36]}$ The non-bonded forces acting on a particle of type $\mathrm{K}$ are calculated from a suitable expression of $V_{\mathrm{K}}$ $(\boldsymbol{r})$ and of its derivatives. In the SCF scheme, a particle interacts with its own surrounding through a mean field. With this assumption, the density-dependent interaction potential $W$ can be written as follows:

$$
\begin{aligned}
W\left[\left\{\phi_{K}(r)\right\}\right]= & \int d r\left(\frac{k_{B} T}{2} \sum_{K K^{\prime}} \chi_{K K^{\prime}} \phi_{K}(\mathbf{r}) \phi_{K^{\prime}}(\mathbf{r})\right. \\
& \left.+\frac{1}{2 \kappa}\left(\sum_{K} \phi_{K}(r)-1\right)^{2}\right)
\end{aligned}
$$

where $\phi_{K}(r)$ is the coarse-grained density of a particle of type $\mathrm{K}$ at position $r$, and $\chi_{K K^{\prime}}$ are the mean field parameters describing the interaction between a particle of type $K$ and the density field due to particles of type $K^{\prime}$. It can be demonstrated, by using the saddle point approximation that the external potential $V_{K}(\boldsymbol{r})$ can be written as:

$$
\begin{aligned}
V_{K}(r) & =\frac{\delta W\left[\phi_{K}(r)\right]}{\delta \phi_{K}(r)} \\
& =k_{B} T \sum_{K^{\prime}} \chi_{K K^{\prime}} \phi_{K^{\prime}}(r)+\frac{1}{\kappa\left(\sum_{K} \phi_{K}(r)-1\right)}
\end{aligned}
$$

The derivation of Equation 2 and the details of the implementation of the MD-SCF approach are reported in the Refs. ${ }^{[36,37]}$ To connect the particles and the field it is crucial to obtain a smooth coarse-grained density function $\phi_{K}(r)$ from the position of the particles. To this aim, and to obtain both the coarse-grained density function and its density derivatives (needed to calculate the forces acting on the particles) a mesh-based approach was used. In a symbolic form this procedure can be written as follows:

$$
\bar{S}\{\widehat{\phi}(r ; \Gamma)\}=\phi(r)
$$

where $\bar{S}$ denotes the mapping from particle position to the coarse-grained density $\phi(r)$ and $\Gamma$ represents the positions of all particles in the system. The density field is obtained by mapping particle positions on a density mesh. In particular, the simulation box is divided into sub-cells. Then, according to the particle positions inside a cell, a fraction of the particle is assigned to each vertex of the sub-cell. A simplified two-dimensional case showing this procedure is reported in panels $\mathrm{B}$ and $\mathrm{C}$ of Scheme 1. A fraction of the particle, proportional to the rectangle areas (panel C of Scheme 1), is assigned to each lattice point. The coarse-grained level of the density is tuned by the mesh size $l$. As example, for a large $l$ value several particles are included in each cell, thus determining a coarser description of the density. Once the coarse-grained density is calculated from the particle coordinates and assigned to all mesh points, the spatial derivatives of the density fields are calculated by numerical interpolation. Then, forces acting on particles can be calculated. In the present work we used the so-called "stencil" method to calculate the density gradient. Such a method is based on the Taylor series developed by Alfaraj et al.-

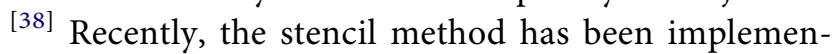
ted in the MD-SCF by Sevink. ${ }^{[39]}$ Further details about the derivation of the potential in the Equation 2 are reported in the Supporting Information, while a complete description of the algorithm used for the interpolation of the density gradient can be found at Ref. ${ }^{[39]}$

Depending on the coarse-grained level of the density, a frequency $\Delta t_{\text {update }}$ is set to update the density field from a new coordinate set at time $t+\Delta t$. For all MD-SCF simulations reported in this work, the frequency update $\Delta t_{\text {update }}$ was set to $0.1 \mathrm{ps,} \mathrm{similarly} \mathrm{to}$ the value used for equivalent systems. ${ }^{[30]}$

\section{Atomistic Model}

The OPLS-AA ${ }^{[40]}$ force-field was used for the traditional MD atomistic simulation, which serves as a reference simulation for the different PP melts. The functional form and parameters of the harmonic bond stretching, angle bending, and torsions can be found in Ref. ${ }^{[40]}$ The intramolecular interactions, including bond, angle, and torsional terms of the MD-SCF model are coincident with those of the OPLS-AA. ${ }^{[40]}$ All bonded and non-bonded parameters are reported in the Supporting Information. 


\section{Computational Details}

All hybrid MD-SCF simulations were performed in the NVT ensemble at the constant temperature of $473 \mathrm{~K}$, which was controlled by the Andersen thermostat ${ }^{[41]}$ with a collision frequency of $7 \mathrm{ps}^{-1}$. Three different mesh sizes $(l=0.2,0.4$, and $0.8 \mathrm{~nm})$ were employed. The time step for particle displacement using velocity Verlet algorithms has been set to $1 \mathrm{fs}$. The OCCAM code $^{[42]}$ was used for all hybrid MD-SCF simulations.

Reference atomistic simulations performed with standard MD technique were carried out in the NPT ensemble by using the GROMACS package (ver. 5.0.1). ${ }^{[43]}$ The temperature was set equal to that of MD-SCF simulations $(T=473 \mathrm{~K})$ and controlled by the Berendsen thermostat $^{[44]}\left(\tau_{T}=0.1 \mathrm{ps}\right)$. The target pressure was held constant at $1 \mathrm{~atm}$ with the Berendsen barostat ${ }^{[44]}\left(\tau_{P}=1\right.$ ps). A cutoff distance of $1.2 \mathrm{~nm}$ was used for both van der Waals and Coulomb interactions. The long-range correction to the electrostatic interactions was calculated using the Ewald summation algorithm. ${ }^{[4]}$ A time step of 2 fs was used for all reference simulations. The bonds involving hydrogen atoms were constrained by using the LINCS algorithm. ${ }^{[46]}$ System compositions and details are reported in Table 1.

\section{Multi-Stage Relaxation Procedure}

The strategy to relax PP melts by MD-SCF approach is based on an increase of the model resolution (tuning the mesh size $l$ ) to equilibrate stepwise by successive equilibrations starting from low (large value of $l$ ) to finer resolutions (small values of $l$ ). The procedure is intended to recover an increasing number of structural correlations from each stage. The advantage of this method is that the model resolution can be tuned simply by changing the mesh size and does not involve the parameterization or reconstruction of the degree of freedom as it is standardly done in coarse-grained model based on particle reduction. ${ }^{[30]}$ The steps of the procedure applied in the

Table 1. System composition of reference MD (50 ns long) and MD-SCF simulations.

\begin{tabular}{lccccc}
\hline System & $\begin{array}{c}\text { No. PP } \\
\text { Chains }\end{array}$ & $\begin{array}{c}\text { No. rep. units/ } \\
\text { chain }\end{array}$ & $\begin{array}{c}\text { Box length } \\
(x=y=z) \\
{[\mathrm{nm}]}\end{array}$ & $\begin{array}{c}\text { Fraction } \\
\text { of } r\end{array}$ & $\begin{array}{c}\text { Fraction } \\
\text { of } r r r\end{array}$ \\
\hline $\begin{array}{c}a-P P- \\
50\end{array}$ & 27 & 50 & 5.2 & 0.5 & - \\
$i$-PP & 27 & 50 & 5.2 & 0 & - \\
s-PP & 27 & 50 & 5.2 & 0.4 & - \\
sPP & 45 & 200 & 9.455 & 0.74 & 0.47 \\
& 45 & 200 & 9.455 & 0.83 & 0.60 \\
& 45 & 200 & 9.455 & 0.87 & 0.71 \\
& 45 & 200 & 9.455 & 0.95 & 0.91 \\
& 45 & 200 & 9.455 & 0.98 & 0.93 \\
\hline
\end{tabular}

present work are listed in the following: (i) The initial configurations of the systems are built by randomly placing the center of mass (c.o.m.) of each PP chain in the simulation box. The box size and the number of chains is chosen according to the experimental density of the PP melt at $473 \mathrm{~K}$. (ii) Three subsequent MD-SCF simulations at increasing density field resolution (reducing the mesh size $l$ from 0.8 to $0.2 \mathrm{~nm}$ ) are performed. In the first stage of the procedure the chains are described by a very coarse density ( $l=0.8 \mathrm{~nm}$, almost 5.3 times larger than the C-C bond distance in the backbone) which allows to equilibrate the chains c.o.m. positions and to pack them in a proper manner (further details in the Supporting Information). The use of coarser density field allows for faster simulations, in terms of both the intrinsic dynamics and the computational effort. ${ }^{[30]}$ (iii) Configurations obtained from the highest density field resolution $(l=$ $0.2 \mathrm{~nm} \sim 1.3$ times the backbone length) are used in the final stage of the procedure where non bonded LennardJones potentials and Coulomb interactions are employed for a short relaxation.

To show in a pictorial way how the mesh size $l$ changes the polymer chain model resolution, the density field, reported as isosurfaces that describe regions with the density $(\rho)>3.5$ atoms $/ \mathrm{nm}^{3}$, is reported in Figure 1. From the figure it is clear that, with a resolution of $l=0.8 \mathrm{~nm}$, the bulkiness of the chain backbone is described, while, at higher resolutions (from $l \leq 0.4 \mathrm{~nm}$ ) the bulkiness of the small $\mathrm{CH}_{3}$ lateral group emerges. In the inset of Figure 1 the details of the density field calculated at $l=0.2 \mathrm{~nm}$ are compared with the atomistic structure. As can be seen, the shape and the details of PP chain reproduced by density field are extremely detailed in comparison with the fullatom resolution.

It is important to underline that no parametrization is needed to generate the well relaxed structures of different polymers by using the described procedure (for homopolymer melt $\chi=0$ ) as reported in the Ref. ${ }^{[30]}$

\section{Results and Discussion}

\section{Short PP Chains}

The first set of systems investigated is composed of $27 \mathrm{PP}$ chains ( $N=50$ monomers, Table 1$)$. Each system is composed of chains characterized by a Bernoullian distribution of diads with the following fractions of racemo $r$ diads: $P_{r}=0$ (isotactic $i$-PP), 0.5 (atactic $a$-PP) and 1.0 (syndiotactic $s$-PP). For each of these systems we performed MD-SCF simulations at constant temperature of $473 \mathrm{~K}$. According to the multi-stage relaxation procedure reported previously, 10 million steps/stage have been 


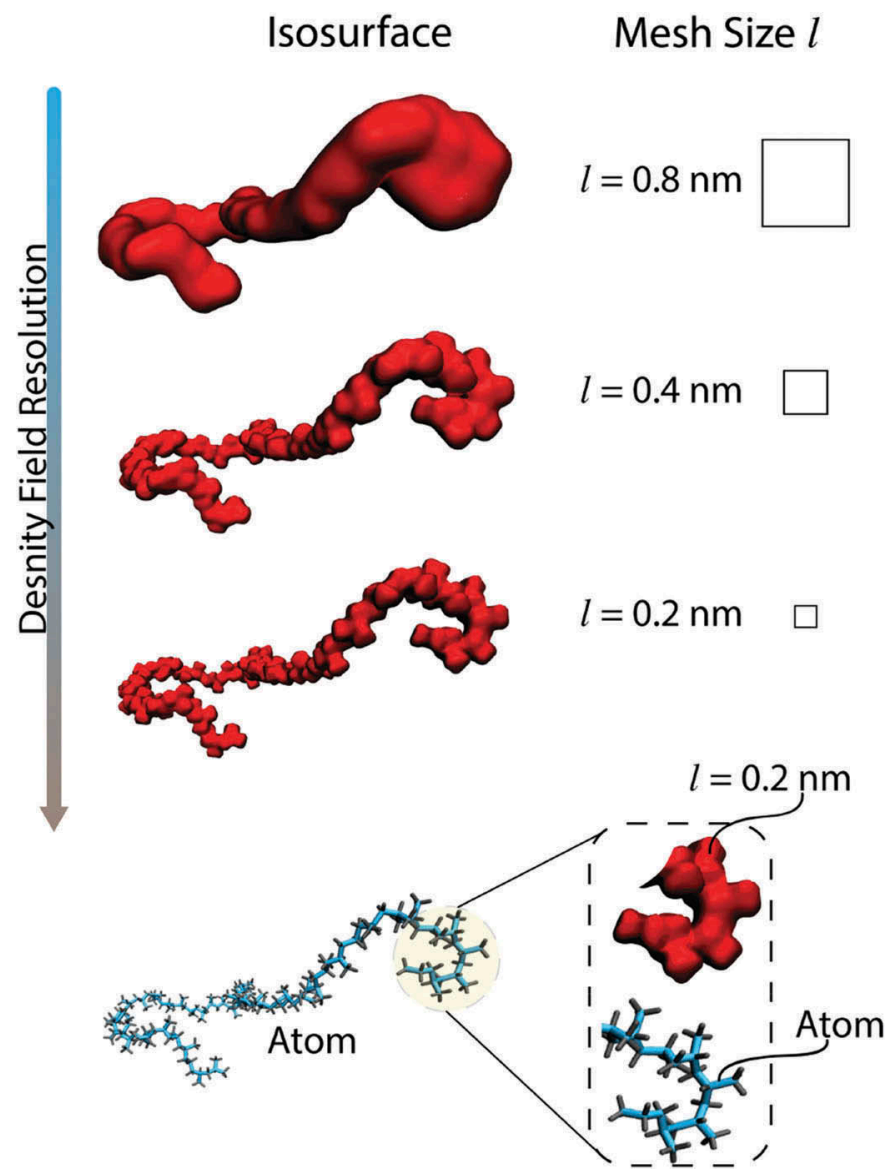

Figure 1. Density isosurfaces calculated from density field generated from a single PP chain $(N=50)$ at different mesh size $(I)$ lengths. The inset showing atom details is reported together with the density isosurface calculate for $I=0.2 \mathrm{~nm}$. All isosurfaces correspond to a density of $\rho>3.5$ atoms $/ \mathrm{nm}^{3}$.

performed for each system and the trajectory produced was used for the analysis.

The stiffness of a polymer chain can be expressed by the Flory's characteristic ratio $C_{n}$ (Equation 4).

$$
C_{n}=\frac{R^{2}}{n l_{b}^{2}}
$$

where $R^{2}$ is the mean-square end-to-end vector distance of PP chains, $n$ is the number of backbone bonds and $l_{b}$ is the bond length $(0.1529 \mathrm{~nm})$. The $C_{n}$ values calculated at each stage of relaxation procedure (different mesh size $l$ ) by using the MD-SCF technique are reported in Table 2. In Figure 2, the calculated $C_{n}$ values from MD-SCF and reference $\mathrm{MD}$ simulations are compared with experimental values measured with SANS reported by Fetters et al. ${ }^{[12,14,15]}$, RIS calculations from Suter, ${ }^{[16,18]}$ Alfonso $^{[47]}$ and Allegra, ${ }^{[48]}$ and MC simulations of a single unperturbed chain reported by Tzounis. ${ }^{[21]}$

As a general behavior, the stiffness of PP chains calculated as Flory's characteristic ratio $C_{n}$ is well
Table 2. $C_{n}$ values for each $P_{m}$ distribution.

\begin{tabular}{lcccc}
\hline System & N (monomer) & $\begin{array}{c}\text { Mesh size I } \\
(\mathrm{nm})\end{array}$ & $P_{r}$ & $C_{n}$ \\
\hline PP & 50 & 0.8 & 0.0 & $3.7(0.2)$ \\
& & & 0.5 & $4.8(0.2)$ \\
& & & 1.0 & $4.1(0.2)$ \\
PP & 50 & 0.4 & 0.0 & $4.2(0.2)$ \\
& & & 0.5 & $4.8(0.2)$ \\
& & & 1.0 & $4.7(0.2)$ \\
PP & \multirow{2}{*}{50} & 0.2 & 0.0 & $5.3(0.1)$ \\
& & & 0.5 & $4.3(0.2)$ \\
& & & 1.0 & $4.5(0.2)$ \\
PP (Reference MD) & 50 & - & 0.0 & $5.8(0.4)$ \\
& & & 0.5 & $6.9(0.2)$ \\
& & & 1.0 & $8.6(0.3)$ \\
\hline
\end{tabular}

reproduced by $\mathrm{MD}$ simulations after the relaxation procedure, based on MD-SCF. This is apparent from the comparison of the results from the last stage of procedure (black square in the figure) with SANS measurements, RIS calculation ${ }^{[16,18,47,48]}$ and MC simulation of single unperturbed chain. ${ }^{[21]}$ The final stage of the relaxation procedure requires approximatively 350 ps 

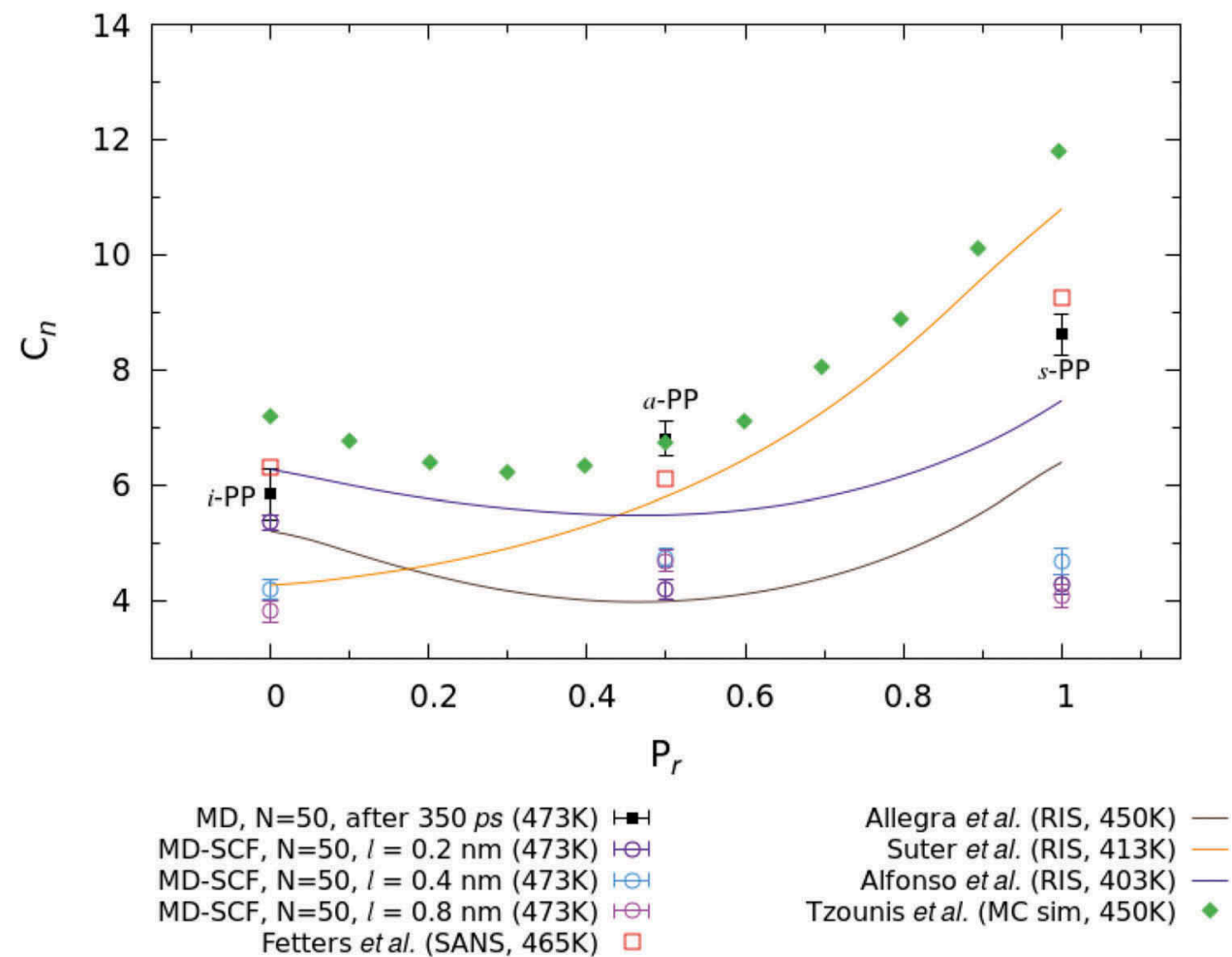

Figure 2. Comparison of $C_{n}$, as function of racemo diad content $P_{r}$, between: MD-SCF simulations $(I=0.2 \mathrm{~nm}$ blue open circle, $I=0.4 \mathrm{~nm}$ light blue circle, $I=0.8 \mathrm{~nm}$ purple blue circle), SANS measurements from Fetters ${ }^{[12,14,15]}$ (open red square), RIS calculation from Alfonso ${ }^{[47]}$ (blue line), Suter ${ }^{[16,18]}$ (orange line), Allegra ${ }^{[48]}$ (dark green line) and MC simulation of single unperturbed chain from Tzounis $^{[21]}$ (green diamonds). The final stage of the relaxation procedure consisting in a short relaxation (350 ps) by MD simulation is reported with filled black square.

to fully allow the $C_{n}$ values to become indistinguishable from long independent MD simulations. In particular, the $C_{n}$ for $a$-PP is slightly stiffer than the experimental value, while the $s$-PP stiffness is slightly lower than SANS data. ${ }^{[12,14,15]}$

By considering the results obtained from the stages of the procedure involving the MD-SCF simulations, no difference between $C_{n}$ values have been found for syndiotactic $(s-\mathrm{PP})$, atactic $(a-\mathrm{PP})$, and isotactic $(i-\mathrm{PP})$ in the whole explored tacticity range (see Figure 2).

In addition to the $C_{n}$ evaluation, the structural correlation reproduced by the MD-SCF model at different resolutions (from coarser $l=0.8 \mathrm{~nm}$ to finer $l=0.2 \mathrm{~nm}$ ) can be evaluated by comparing the backbone-backbone intramolecular radial distribution function (RDF) with the reference MD simulations (see Figure 3).

As can be seen from Figure 3, the intermolecular RDF of backbone-backbone with mesh size $l=0.8 \mathrm{~nm}$ does not match reference MD simulations at low $r$ values. Instead, with a mesh size $l \leq 0.4 \mathrm{~nm}$ (see panel B, C, D, of Figure 3) the RDFs gain more details approaching to the correlation obtained in standard MD simulations, as found also in several previous works. ${ }^{[28,30-32,34,49]}$ However, the small difference between the RDF calculated for different tacticity, which is kept by reference MD simulation and in agreement with previous studies, ${ }^{[50]}$ is not completely reproduced by MD-SCF simulations (see panel A of Figure 3). The larger deviation from MD is observed for $s$-PP (panel B of Figure 3). In particular, with the mesh size $l=0.2 \mathrm{~nm}$, the position of first peak of RDF is shifted at higher $r$ values of $\sim 0.2 \mathrm{~nm}$ with respect to the MD reference simulation. In case of $\mathrm{CH}_{3}$ substituent group, the RDFs calculated from MD-SCF simulations with a mesh size $l \leq 0.4 \mathrm{~nm}$ show similar correlations for different tacticity (Figure $4 \mathrm{~b}-\mathrm{d}$ ), as also found in the case of reference MD simulations (panel A of Figure 4).

In both cases, i.e., backbone-backbone and $\mathrm{CH}_{3}-\mathrm{CH}_{3}$, the first and most intense peak in the RDF distributions has a very local nature and can be recovered only using a pair potential. We demonstrated that a short relaxation is required to fully recover this local pair correlation. ${ }^{[28]}$ In fact, as reported in Figure 5, a relaxation time of about 70 ps is enough to obtain indistinguishable RDF distributions for the backbone-backbone pair for all investigated tacticity range. In particular, starting from the last configuration of MD-SCF with mesh size $l=0.2 \mathrm{~nm}$, the longest relaxation 

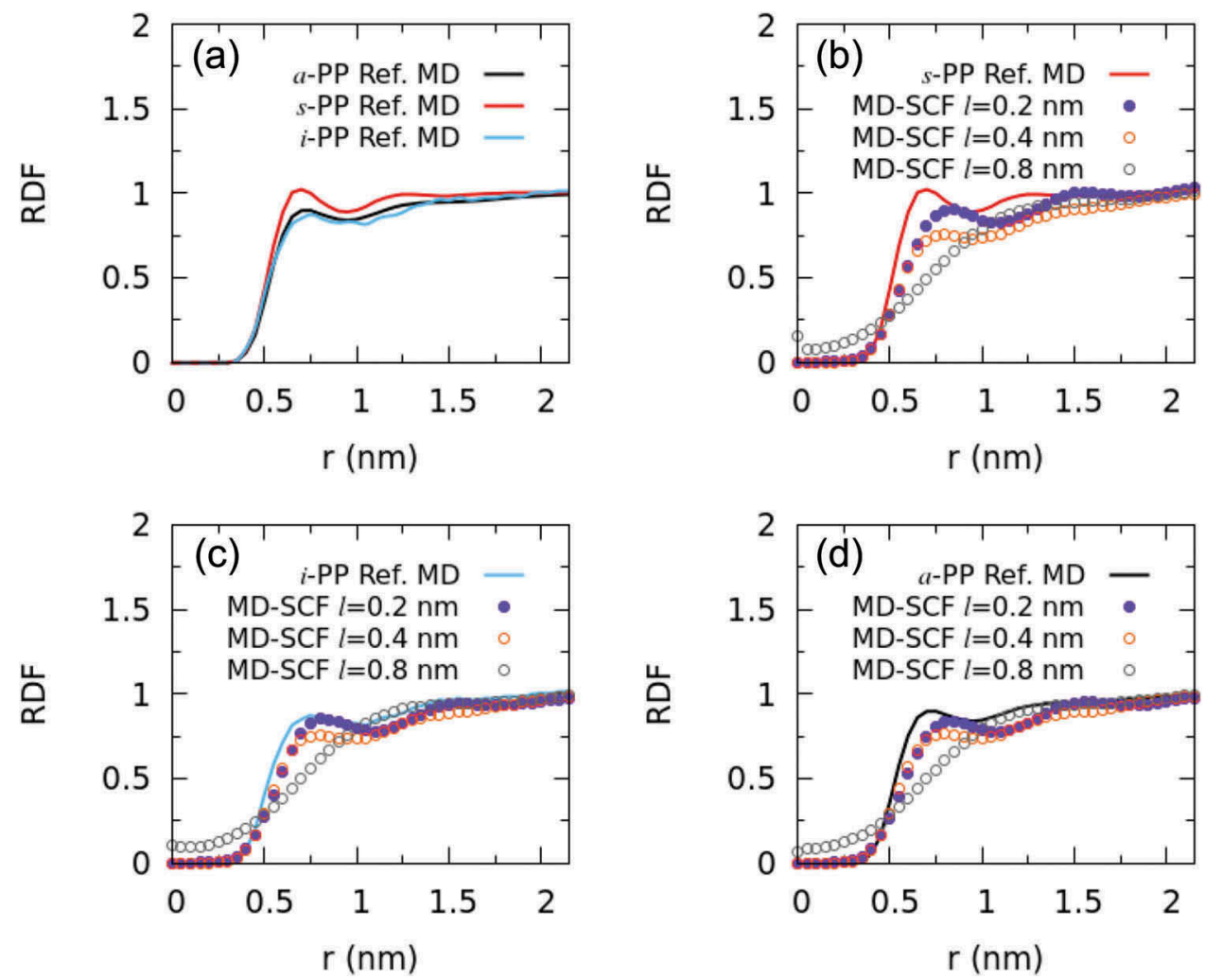

Figure 3. Comparison of backbone-backbone intermolecular RDF distribution: (a) Reference MD simulations for s-PP (red line), $a$-PP (black line) and $i$-PP (light blue line). (b) Reference MD of $s$-PP versus MD-SCF simulations. (c) Reference MD of $i$-PP versus MD-SCF simulations. (d) Reference MD of $a$-PP versus MD-SCF simulations.
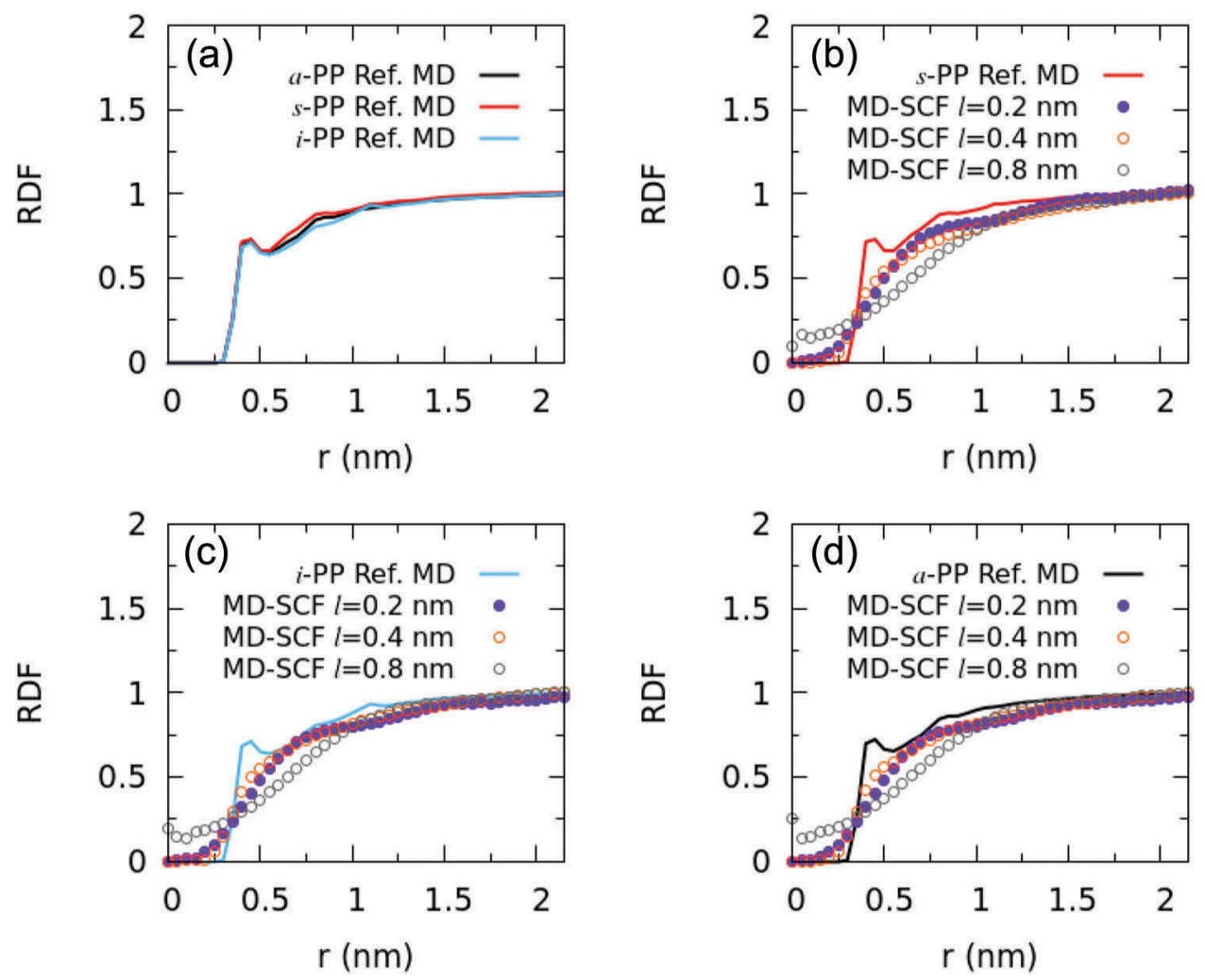

Figure 4. Comparison of $\mathrm{CH}_{3}-\mathrm{CH}_{3}$ intermolecular RDF distribution: (a) Reference MD simulations for s-PP (red line), $a$-PP (black line), and $i$-PP (light blue line). (b) Reference MD of s-PP versus MD-SCF simulations. (c) Reference MD of $i$-PP versus MD-SCF simulations. (d) Reference MD of $a$-PP versus MD-SCF simulations. 

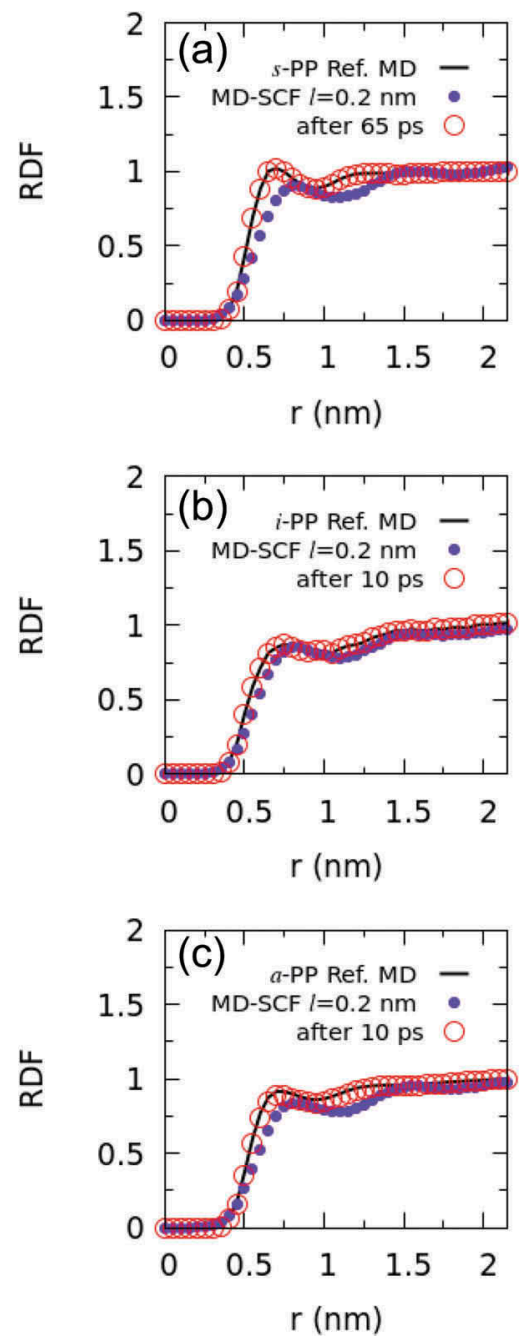

Figure 5. Comparison of intermolecular RDF for the backbonebackbone pair between reference MD (black line), MD-SCF (blue circle), and after: (a) 65 ps for s-PP, (b) 10 ps for $i-P P$, (c) 10 ps for $a$-PP.

time of 65 ps is required in case of $s$-PP (see panel A of Figure 5), while, 10 ps are sufficient for $a$-PP and $i$-PP (see panel $\mathrm{B}$ and $\mathrm{C}$ of Figure 5). For $\mathrm{CH}_{3}-\mathrm{CH}_{3}$, a relaxation time of $10 \mathrm{ps}$ is sufficient to fully recover the short-range correlations (more details in the Supporting Information).

According to the results discussed in this section, it is possible to obtain for the proposed procedure results practically equivalent to models based on traditional MD simulations without the limitations related to the slow relaxation times.

\section{Long PP Chains}

In order to study and to properly include the effects of tacticity in our models it is necessary to consider high molecular weight (M.W.) models for different reasons.
The main reason is that, to model highly stereoregular (but not fully stereoregular) polymer chains, as for example a high fraction of diads $[r]$ and pentads $[\mathrm{rrrr}]$ close to one but not fully syndiotactic, a long pseudo asymmetric center sequence (and hence a high molecular weight) is needed. The MD-SCF method well fits these requirements due to the fact that the mean-field approximations are more accurate for polymer systems with large molecular weights. ${ }^{[30]}$ In particular, in addition to the mesh size $l$ and density update frequency, the fluctuation of the collective density depends on the invariant degree of polymerization $\tilde{N}=\left(\rho_{0} b^{3}\right)^{2} N$, where $\rho_{0}$ is the average number density of repeating units, $b$ is the Kuhn length and $N$ is the number of monomers. In the limit of $\tilde{N} \rightarrow \infty$ the collective density fluctuations are suppressed leading to an increase in the accuracy of the approximations in the SCF theory. ${ }^{[30,51,52]}$ This means that upon increasing the monomer number $N$, the accuracy of the MD-SCF simulations increases in turn. Last but not least, MD-SCF allows to efficiently relax polymer melts with high molecular weights, which is practically not feasible or extremely hard by using atomistic MD simulations. For example, it is extremely difficult to move the c.o.m. of polymer chains $(N=200)$ at least of their radius of gyration by using traditional MD simulations (see Supporting Information).

For all these reasons, the second set of systems are composed of long $s$-PP chains (45 chains of $N=200$ monomers, Table 1) with high tacticity. In particular, five increasing racemo diads fraction $\left(\mathrm{P}_{r}\right)$ have been chosen: $0.74,0.83,0.87,0.95$, and 0.98 , respectively. For each $\mathrm{P}_{r}$ fraction, five different distributions (according to the Bernoulli probability distribution) were generated to build the PP chains. In total, each system contains $45 s$-PP chains with 5 independent racemo diads distributions according to the fixed values of $\mathrm{P}_{r}$. A Bernoullian probability distribution of the diads was generated with the algorithm described by Ladd. ${ }^{[53]}$

The MD-SCF simulations of the second set of systems were performed at constant temperature of $473 \mathrm{~K}$. As in the short chain case, the resolution of the coarse grain density was increased step by step (from a mesh size of $l=0.8$ to 0 . $\mathrm{nm}$ and from 0.4 to $0.2 \mathrm{~nm}$ ). In Figure 6, the stiffness of the $s$-PP chains has been calculated through the $C_{n}$ parameter and compared with MD-SCF simulations results with $N=$ 50 , experimental and theoretical behaviors.

As it can be seen from Figure 6, the MD-SCF simulations of higher molecular weight reproduce, better than those with $N=50$, the main trend of $C_{n}$ which increases with increasing $\mathrm{P}_{r}$ as predicted by theoretical RIS model of Allegra, ${ }^{[48]}$ Suter ${ }^{[16,18]}$ and Alfonso ${ }^{[47]}$ and observed experimentally by Fetters. ${ }^{[12,14,15]}$ In particular, the MD-SCF 

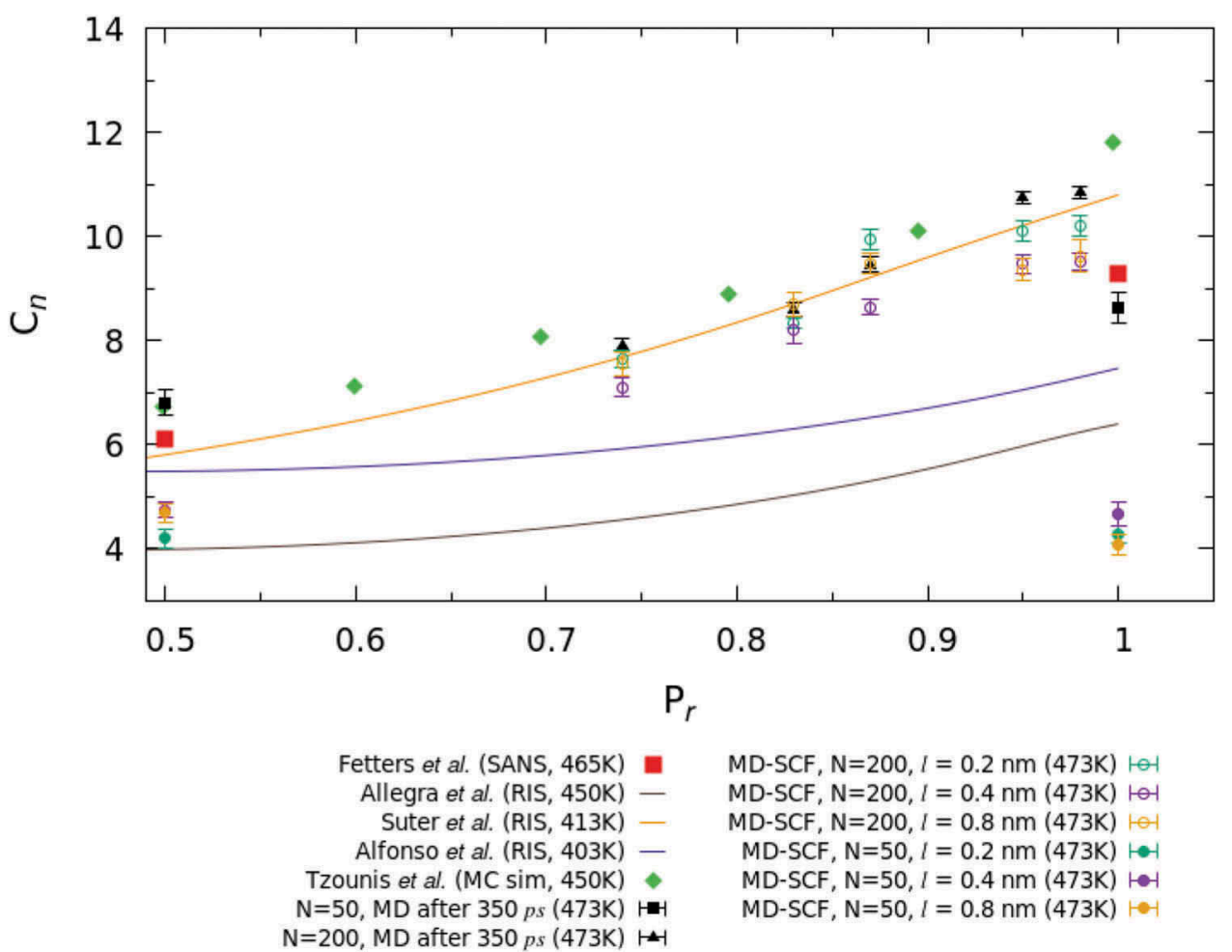

Figure 6. Comparison of Flory's Characteristic ratio $C_{n}$ as function of racemo diad content $P_{r}$, between: MD-SCF simulations of $s$-PP with $I=0.2 \mathrm{~nm}(N=200$ blue open circle, $N=50$ blue circle), $I=0.4 \mathrm{~nm}(N=200$ light blue open circle, $N=50$ light blue circle), $I=0.8 \mathrm{~nm}\left(N=200\right.$ yellow open circle, $N=50$ yellow circle). SANS measurements from Fetters ${ }^{[12,14,15]}$ (open red square). RIS calculation from Alfonso ${ }^{[47]}$ (blue line), Suter ${ }^{[16,18]}$ (orange line), Allegra ${ }^{[48]}$ (dark green line). MC simulation of single unperturbed chain from Tzounis ${ }^{[21]}$ (green diamonds). Last stage of relaxation procedure for $N=50$ (black square) and $N=200$ (black triangle).

simulation with $l=0.8 \mathrm{~nm}$ (yellow open circle in the figure) follows the same monotonic increase of $C_{n}$ versus $\mathrm{P}_{n}$ while, both MD-SCF simulations with $l \leq 0.4 \mathrm{~nm}$ show a deviation from the monotonic behavior at $\mathrm{P}_{r}=0.87$. More in general, the stiffness of $s$-PP chains we calculated is close to the results of Suter (RIS at $413 \mathrm{~K}$, yellow line in Figure 6) and experimental SANS measurements at $465 \mathrm{~K}^{[12,14,15]}$ and slightly lower than the estimation of Tzounis et al. ${ }^{[21]} \mathrm{calcu}^{-}$ lated from single unperturbed chain MC simulations at $450 \mathrm{~K}$. The final stage of the relaxation procedure, which requires a short relaxation with standard $\mathrm{MD}$ simulation, in case of $N=200$ gives results (black triangle) very close to those ones obtained from the different stage involving the MD-SCF simulation. More in general, the stiffness of the $\mathrm{s}$-PP chains is better reproduced from MD-SCF simulations with larger $N$. As mentioned before, this can be attributed to the different value of the invariant degree of polymerization $\tilde{N}$ which is $1.1 \times 10^{4}$ for $N=50$ and $5.6 \times 10^{4}$ for $N=200$.

To analyze in more detail the $s$-PP chain conformations we computed the distribution of torsional angles $(\phi)$ belonging to the backbone of polymer chains for each $\mathrm{P}_{r}$ (See the Supporting Information). Then, we identified the trans $(t)$ and gauche $(g)$ torsional states at $180^{\circ}$ and $78^{\circ}$, respectively.
In panel A of Figure 7 we report a comparison of the average fractions of trans $\left(\mathrm{P}_{t}\right)$ and gauche $\left(\mathrm{P}_{g}\right)$ state at increasing $\mathrm{P}_{r}$ content. In particular, a qualitative agreement between our calculations and the results of Tzounis ${ }^{[21]}$ calculated from MC simulations of a single unperturbed chain, emerges from the comparison. In addition, we reported in panel $\mathrm{B}$ of the same figure the maximum length of trans sequence defined as the number of consecutive torsional $\phi$ angles in the backbone corresponding to trans state. As expected, moving from relatively lower to higher $\mathrm{P}_{r}$ fraction the maximum length of trans sequence becomes higher (from 17 to 22). In Figure $7 c$, the $X$-ray scattering calculated from simulations of PP ( $=50$ and 200) systems, relaxed by using the described procedure, are compared with the experimental data. ${ }^{[50]}$ Likely, due to the larger molecular weight of $s$-PP $\left(\mathrm{N}=200\right.$ ), the region at low $q$ (from 2 to $5 A A^{-1}$ ) is slightly better reproduced.

As mentioned in the Introduction, the relation between the microstructure of a polymer (tacticity) and the macroscopic parameters of a polymer melt have been experimentally well studied. To this aim, SANS and plateau modulus $\left(G_{N}^{0}\right)$ measurements have been used. ${ }^{[10,14,15]}$ Recently, Ahmad et al. reported a detailed 

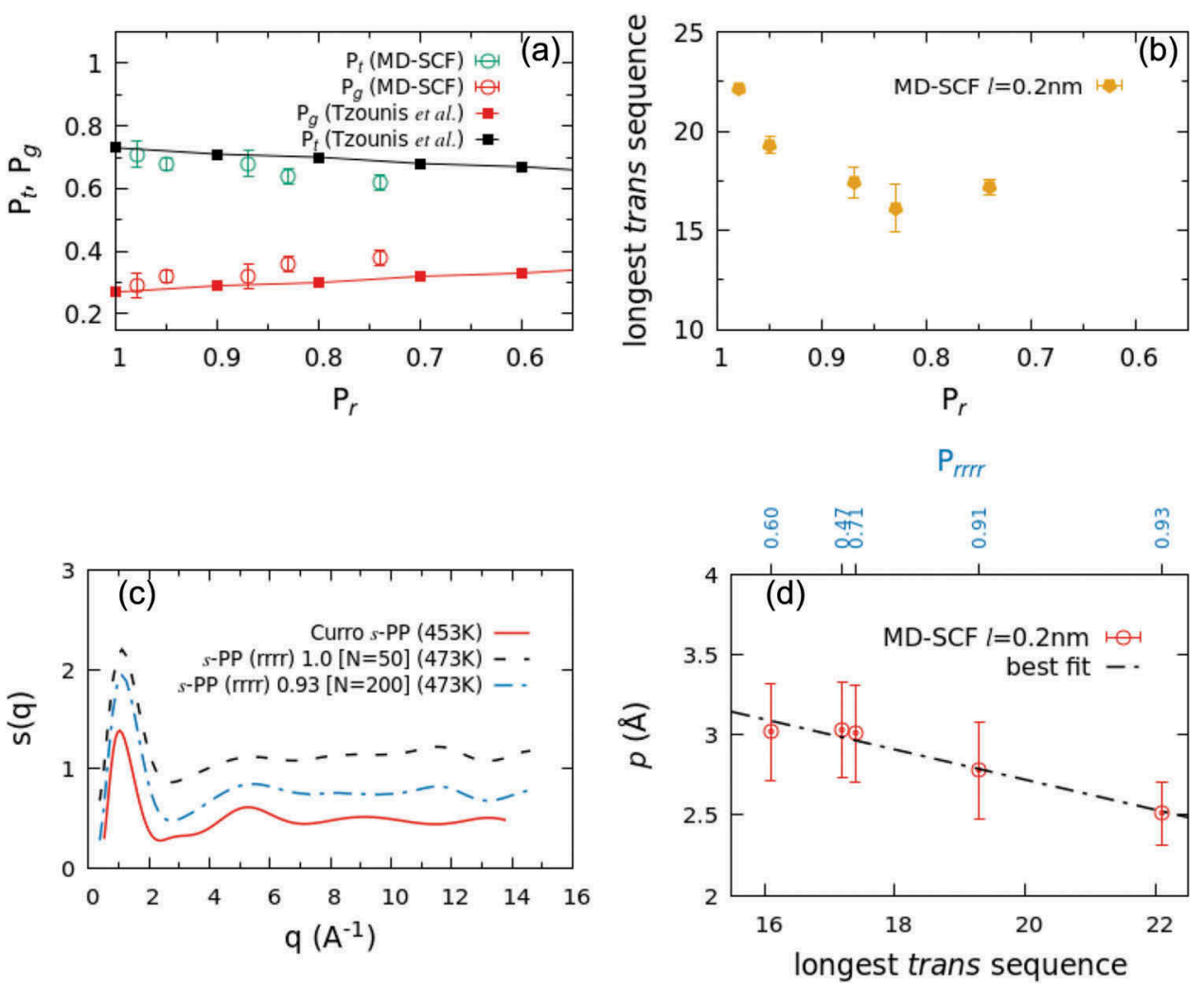

Figure 7. (a) Average of trans $\left(\mathrm{P}_{t}\right)$ and gauche $\left(\mathrm{P}_{g}\right)$ probability as function of $\mathrm{P}_{r}$ fraction. (b) Maximum length of trans sequence calculated from MD-SCF simulations as function of $P_{r}$. All data referred to MD-SCF simulation have been calculated on systems with a mesh size $I=0.2 \mathrm{~nm}$. (c) Scattering function $s(q)$ calculated for different chain length $s$-PP are compared with Experimental behavior $^{[50]}$ (red line). The Scattering functions have been calculated on the relaxed MD simulation by averaging configurations over the last 2 ns. (d) longest trans sequence, for each tacticity of s-PP, is reported as function of the packing length $p$ (red open circle). The segmented black line is the best linear fitting $(y=a+b x, a=4.6 \dot{\mathrm{A}}$, and $b=-0.094 \dot{\mathrm{A}} /$ transseq.).

study on the relationship between high stereoregular $s$-PP melts and the viscoelasticity of the polymer. ${ }^{[10]}$ Using single-center metallocene and half-metallocene catalysts they synthetized s-PP samples characterized by different content of $[\mathrm{rrrr}]$ pentads and investigated their viscoelastic properties. In addition, in the work of Ahmad et al. the packing length $(p)$ was estimated by using the plateau modulus $G_{N}^{0}$. They found that the higher is the content of racemo pentads [ $r r r r]$ the smaller is the packing length $p$. It is worth noting that a low value of the packing length value corresponds to a chain segment which occupies less volume. ${ }^{[54]}$

As a test case, we applied our method to calculate, and compare with experiments, the values of the packing length calculated on polymer melts having the same experimental pentad fraction reported by Ahmad et al. (see the pentad fraction in Table 1).

The packing length $(p)$ is defined as the ratio between the effective volume of the polymer chain and its mean square end-to-end distance. ${ }^{[55]}$ From rheological measurements, $p$ can be calculated through the Equation 5:

$$
p=\sqrt[3]{\left(\frac{A^{2} k T}{864 G_{N}^{0}}\right)}
$$

where $A^{2}$ is a constant independent on temperature ( $A=1.545), k$ is the Boltzmann constant, $T$ is the absolute temperature, and $G_{N}^{0}$ is the plateau modulus of the polymer.

An equivalent method to calculate $p$ which is more suitable for the quantities computed from MD simulations is reported in the Equation 6:

$$
p=\frac{M}{R^{2} \rho N_{A}}
$$

where $M$ is the mass of the polymer chain, $R$ is the endto-end vector distance, $\rho$ is the mass density of the system, and $N_{A}$ is the Avogadro number.

In order to have a direct comparison between the experimental and calculated data, the same experimental samples with high racemo pentad fraction of $0.93,91.5$, $70.6,60.1,46.9$ of $[\mathrm{rrrr}]$ were generated for $s$-PP with $N=$ 200 (see the Table 1). Starting from the last configuration of each MD-SCF simulation $(l=0.2 \mathrm{~nm})$ performed in 
NVT ensemble, a short relaxation (1 ns in NVT ensemble) with standard MD has been performed. Then, NPT simulations long enough to have equilibrated densities were performed at same experimental temperature $\mathrm{T}=$ $473 \mathrm{~K}$. From those simulations, the equilibrium end-toend distance and density values needed to compute the packing length have been calculated. In Table 3 experimental and calculated $p$ (with Equation 6) are reported.

We found that $p$ values calculated from the MD systems, equilibrated by applying the procedure described previously, are in good agreement with the experimental values measured through plateau modulus $G_{N}^{0}{ }^{\left[{ }^{[10]}\right.}$ As a general behavior, the increase of the pentad content rrr causes a decrease of the packing length. In particular, going from $[\mathrm{rrrr}]=0.47$ to $0.93 p$ decreases from 3 to 2.5 , similarly to the experimental trend reported by Ahmad et al. ${ }^{[10]}$, which shows a decrease of $p$ from 3.4 to 2.8 . This result confirms that the MD-SCF method is suitable to model fine structural differences due to the polymer microstructure. Moreover, similar trends have been measured on high stereoregular polypropylene, with different racemo diad fraction, by Liu ${ }^{[56]}$ and Fetters. ${ }^{[57]}$ We argue that the decrease of packing length upon increasing the rrr content is a direct consequences of the more compact average set of accessible chain conformations as indicated by the simultaneous increase of the maximum length of trans sequences (Figure 7b). Moreover, we observe a linear correlation between the increase of the longest trans sequence and the packing length $p$ as reported in panel D of Figure 7.

\section{Conclusions}

The MD-SCF approach has been tested in the reproduction of the effect of tacticity on the structural properties of PP melts by applying a multi-stage relaxation procedure validated in a previous work. ${ }^{[30]}$ We propose a systematic study, by using MD-SCF simulations, investigating two set of systems. In the first set atactic, isotactic, and syndiotactic PP chains of low M.W. have been considered. We applied a relaxation procedure which successfully reproduces the stiffness of the PP chains, in terms of $C_{n}$, with equal and indistinguishable accuracy with respect to independent MD simulations.

Table 3. Experimental ${ }^{[10]}$ and calculated $p$ values.

\begin{tabular}{lccc}
\hline System & {$[r r r]$ fraction } & $\begin{array}{c}p \text { (calc.) } \\
(\AA)\end{array}$ & $\begin{array}{c}p \text { (exp.) } \\
(\AA)\end{array}$ \\
\hline s-PP $(\mathrm{N}=200)$ & 0.93 & $2.51(0.2)$ & 2.83 \\
& 0.91 & $2.78(0.3)$ & 3.04 \\
& 0.71 & $3.01(0.3)$ & 3.26 \\
& 0.60 & $3.02(0.3)$ & 3.34 \\
& 0.47 & $3.03(0.3)$ & 3.39 \\
\hline
\end{tabular}

In the second set of simulations, a higher $\mathrm{M}$. W. of $s$-PP has been investigated. In particular, syndiotactic PP chains $(\mathrm{N}=200)$ were generated with an increased fraction of racemo diads (according to Bernoullian distribution) going from 0.74 to 0.98 . From MD-SCF simulations we found that the stiffness of the polymer is well reproduced with respect to SANS measurements, RIS predictions and MC simulations of a single unperturbed chain. In addition, the results from MD-SCF simulations indicate that even with a lower resolution of the coarse grain density (which can be easily controlled by changing the mesh size $l$ ) the stiffness of $s$-PP chains, and hence the excluded volume of polymer chains, shows a good match with experiments. This result is not surprising since the approximations of the MD-SCF approach gain accuracy at high M.W., as proved in a previous work. ${ }^{[30]}$

Since the MD-SCF approach is capable to reproduce fine structural differences as a function of the tacticity, we reported a first application studying the packing length of very highly stereoregular syndiotactic PP. To this aim, we generated chains with a Bernoullian distribution of pentads ( $\mathrm{rrr}$ ) equal to experimental samples reported by Ahmad et al. ${ }^{[10]}$ Applying the relaxation procedure on the systems with high stereoregularity, and performing a short relaxation by standard MD simulations, we computed and compared the packing length for all different pentad fractions. We found a very good agreement between the calculated packing length and those estimated from rheological measurements.

The present study expands the field of applicability of MD-SCF method at atomistic resolution to the study of stereoregular polymeric materials, in particular to the description and understanding of the effects of fine microstructural features of vinyl polymers on structural properties.

\section{Acknowledgments}

The computer resources and related technical support used for this work have been provided by CRESCO/ENEAGRID High Performance Computing Infrastructure and its Staff. CRESCO/ ENEAGRID High Performance Computing Infrastructure is funded by ENEA, the Italian National Agency for New Technologies, Energy and Sustainable Economic Development and by Italian and European research programs.

\section{ORCID}

Antonio De Nicola (1) http://orcid.org/0000-0002-9581-6226 


\section{References}

[1] Flory, P. J. On the Stereochemical Constitution and Nuclear Magnetic Resonance Spectra of Polypropylenes. Macromolecules. 1970, 3, 613-617. DOI: 10.1021/ ma60017a601.

[2] Stehling, F. C. Stereochemical Configurations of Polypropenes by High Resolution Nuclear Magnetic Resonance. J. Polym. Sci. Part A Gen. Pap. 1964, 2, 1815-1823. DOI: 10.1002/pol.1964.100020422.

[3] Callan, J. F.; Fawcett, A. H.; Malcolm, R. K. The Effect of Tacticity on the Conformational Properties of Poly (1-olefin Sulfone)s. J. Polym. Res. 2008, 15, 107-113. DOI: $10.1007 / \mathrm{s} 10965-007-9149-\mathrm{z}$.

[4] Moore, E. P. Polypropylene Handbook: Polymerization, Characterization, Properties, Processing, Applications; Hanser, Cincinnati, 1996.

[5] Fink, J. K. Reactive Polymers: Fundamentals and Applications: A Concise Guide to Industrial Polymers; Plastics Design Library Elsevier Science, U.S., 2017.

[6] Geng, K.; Tsui, O. K. C. Effects of Polymer Tacticity and Molecular Weight on the Glass Transition Temperature of Poly(methyl Methacrylate) Films on Silica. Macromolecules. 2016, 49, 2671-2678. DOI: 10.1021/acs.macromol.6b00108.

[7] Madkour, T. M.; Mark, J. E. Modeling of the Crystallization of Isotactic Polypropylene Chains. J. Polym. Sci. Part B Polym. Phys. 1997, 35, 2757-2764. DOI: 10.1002/(ISSN)1099-0488.

[8] Arrighi, V.; Batt-Coutrot, D.; Zhang, C.; Telling, M. T. F.; Triolo, A. Effect of Tacticity on the Local Dynamics of Polypropylene Melts. J. Chem. Phys. 2003, 119, 1271-1278. DOI: 10.1063/1.1579476.

[9] Eckstein, A.; Suhm, J.; Friedrich, C.; Maier, R. D.; Sassmannshausen, J.; Bochmann, M.; Mülhaupt, R. Determination of Plateau Moduli and Entanglement Molecular Weights of Isotactic, Syndiotactic, and Atactic Polypropylenes Synthesized with Metallocene Catalysts. Macromolecules. 1998, 31, 1335-1340. DOI: 10.1021/ma971270d.

[10] Ahmad, N.; Di Girolamo, R.; Auriemma, F.; De Rosa, C.; Grizzuti, N. Relations between Stereoregularity and Melt Viscoelasticity of Syndiotactic Polypropylene. Macromolecules. 2013, 46, 7940-7946. DOI: 10.1021/ ma401469a.

[11] Chile, L. E.; Mehrkhodavandi, P.; Hatzikiriakos, S. G. A Comparison of the Rheological and Mechanical Properties of Isotactic, Syndiotactic, and Heterotactic Poly(lactide). Macromolecules. 2016, 49, 909-919. DOI: 10.1021/acs.macromol.5b02568.

[12] Zhongde, X.; Hadjichristidis, N.; Carella, J. M.; Fetters, L. J. Characteristic Ratios of Atactic Poly(vinylethylene) and Poly(ethylethylene). Macromolecules. 1983, 16, 925-929. DOI: 10.1021/ ma00240a019.

[13] Xu, Z.; Hadjichristidis, N.; Fetters, L. J.; Mays, J. W. Structure/chain-flexibility Relationships of Polymers BT - Physical Properties of Polymers; Springer Berlin Heidelberg: Berlin, Heidelberg, 1995; pp 1-50.

[14] Fetters, L. J.; Graessley, W. W.; Krishnamoorti, R.; Lohse, D. J. Melt Chain Dimensions of Poly(ethylene -1-butene) Copolymers via Small Angle Neutron
Scattering. Macromolecules. 1997, 30, 4973-4977. DOI: $10.1021 / \operatorname{ma} 961408 \mathrm{c}$.

[15] Smith, G. D.; Yoon, D. Y.; Jaffe, R. L.; Colby, R. H.; Krishnamoorti, R.; Fetters, L. J. Conformations and Structures of Poly(oxyethylene) Melts from Molecular Dynamics Simulations and Small-Angle Neutron Scattering Experiments. Macromolecules. 1996, 29, 3462-3469. DOI: 10.1021/ma951621t.

[16] Suter, U. W.; Flory, P. J. Conformational Energy and Configurational Statistics of Polypropylene. Macromolecules. 1975, 8, 765-776. DOI: 10.1021/ ma60048a018.

[17] Boyd, R. H.; Breitling, S. M. Conformational Properties of Polypropylene1. Macromolecules. 1972, 5, 279-286. DOI: $10.1021 / \mathrm{ma} 60027 \mathrm{a} 010$.

[18] Theodorou, D. N.; Suter, U. W. Shape of Unperturbed Linear Polymers: Polypropylene. Macromolecules. 2002, 18, 1206-1214. DOI: 10.1021/ma00148a028.

[19] Antoniadis, S. J.; Samara, C. T.; Theodorou, D. N. Molecular Dynamics of Atactic Polypropylene Melts. Macromolecules. 1998, 31, 7944-7952. DOI: 10.1021/ ma9807318.

[20] Antoniadis, S. J.; Samara, C. T.; Theodorou, D. N. Effect of Tacticity on the Molecular Dynamics of Polypropylene Melts. Macromolecules. 1999, 32, 8635-8644. DOI: 10.1021/ma990888f.

[21] Tzounis, P. N.; Argyropoulou, D. V.; Anogiannakis, S. D.; Theodorou, D. N. Tacticity Effect on the Conformational Properties of Polypropylene and Poly(ethylene-propylene) Copolymers. Macromolecules. 2018, 51, 6878-6891. DOI: 10.1021/acs.macromol.8b01099.

[22] Fritz, D.; Harmandaris, V. A.; Kremer, K.; Van Der Vegt, N. F. A. Coarse-grained Polymer Melts Based on Isolated Atomistic Chains: Simulation of Polystyrene of Different Tacticities. Macromolecules. 2009, 42, 7579-7588. DOI: 10.1021/ma901242h.

[23] Chiessi, E.; Paradossi, G. Influence of Tacticity on Hydrophobicity of Poly(N-isopropylacrylamide): A Single Chain Molecular Dynamics Simulation Study. J. Phys. Chem. B. 2016, 120, 3765-3776. DOI: 10.1021/acs.jpcb.6b01339.

[24] Lu, K. T.; Tung, K. L. Molecular Dynamics Simulation Study of the Effect of PMMA Tacticity on Free Volume Morphology in Membranes. Korean J. Chem. Eng. 2005, 22, 512-520. DOI: 10.1007/BF02706635.

[25] Noorjahan, A.; Choi, P. Thermodynamic Properties of Poly(vinyl Alcohol) with Different Tacticities Estimated from Molecular Dynamics Simulation. Polymer (Guildf). 2013, 54, 4212-4219. DOI: 10.1016/ j.polymer.2013.05.073.

[26] Soldera, A. Energetic Analysis of the Two PMMA Chain Tacticities and PMA through Molecular Dynamics Simulations. Polymer (Guildf). 2002, 43, 4269-4275. DOI: 10.1016/S0032-3861(02)00240-9.

[27] Baschnagel, J.; Binder, K.; Doruker, P.; Gusev, A. A.; Hahn, O.; Kremer, K.; Mattice, W.L.; Müller-Plathe, F.; Murat, M.; Paul, W.; et al. Bridging the Gap between Atomistic and Coarse-Grained Models of Polymers: Status and Perspectives. Viscoelasticity At. Model. Stat. Chem. 2000, 152, 41-156.

[28] De Nicola, A.; Kawakatsu, T.; Müller-Plathe, F.; Milano, G. Fast Relaxation of Coarse-grained Models 
of Polymer Interphases by Hybrid Particle-field Molecular Dynamics: Polystyrene-silica Nanocomposites as an Example. Eur. Phys. J. Spec. Top. 2016, 225, 1817-1841. DOI: 10.1140/epjst/e201660127-0.

[29] Milano, G.; Müller-Plathe, F. Mapping Atomistic Simulations to Mesoscopic Models: A Systematic Coarse-Graining Procedure for Vinyl Polymer Chains. J. Phys. Chem. B. 2005, 109, 18609-18619. DOI: 10.1021/jp0523571.

[30] De Nicola, A.; Kawakatsu, T.; Milano, G. Generation of Well-relaxed All-atom Models of Large Molecular Weight Polymer Melts: A Hybrid Particle-continuum Approach Based on Particle-field Molecular Dynamics Simulations. J. Chem. Theory Comput. 2014, 10, 5651-5667. DOI: 10.1021/ct500492h.

[31] Munaò, G.; Pizzirusso, A.; Kalogirou, A.; De Nicola, A.; Kawakatsu, T.; Müller-Plathe, F.; Milano, G. Molecular Structure and Multi-body Potential of Mean Force in Silica-polystyrene Nanocomposites. Nanoscale. 2018, 10, 21656-21670. DOI: 10.1039/C8NR05135F.

[32] De Nicola, A.; Avolio, R.; Della Monica, F.; Gentile, G.; Cocca, M.; Capacchione, C.; Errico, M. E.; Milano, G. Rational Design of Nanoparticle/monomer Interfaces: A Combined Computational and Experimental Study of in Situ Polymerization of Silica Based Nanocomposites. RSC Adv. 2015, 5, 71336-71340. DOI: 10.1039/C5RA13154E.

[33] Zhao, Y.; Byshkin, M.; Cong, Y.; Kawakatsu, T.; Guadagno, L.; De Nicola, A.; Yu, N.; Milano, G.; Dong, B. Self-assembly of Carbon Nanotubes in Polymer Melts: Simulation of Structural and Electrical Behaviour by Hybrid Particle-field Molecular Dynamics. Nanoscale. 2016, 8, 15538-15552. DOI: 10.1039/C6NR03304K.

[34] De Nicola, A.; Correa, A.; Milano, G.; La Manna, P.; Musto, P.; Mensitieri, G.; Scherillo, G. Local Structure and Dynamics of Water Absorbed in Poly(ether Imide): A Hydrogen Bonding Anatomy. J. Phys. Chem. B. 2017, 121, 3162-3176. DOI: 10.1021/acs. jpcb.7b00992.

[35] De Nicola, A.; Kawakatsu, T.; Milano, G. Simulations. J. Chem. Theory. Comput. 2014, 10, 5651-5667. DOI: $10.1021 /$ ct500492h.

[36] Milano, G.; Kawakatsu, T. Hybrid-MD-SCF. J. Chem. Phys. 2009, 130, 184904.

[37] Pressure, I.; Back, G. Pressure Calculation. J. Chem. Phys. 2016, 133, 1-3.

[38] Alfaraj, M.; Wang, Y.; Luo, Y. Enhanced Isotropic Gradient Operator. Geophys. Prospect. 2014, 62, 507-517. DOI: 10.1111/gpr.2014.62.issue-3.

[39] Sevink, G. J. A.; Schmid, F.; Kawakatsu, T.; Milano, G. Combining Cell-based Hydrodynamics with Hybrid Particle-field Simulations: Efficient and Realistic Simulation of Structuring Dynamics. Soft Matter. 2017, 13, 1594-1623. DOI: 10.1039/C6SM02252A.

[40] Jorgensen, W. L.; Mcdonald, N. A. Development of an All-atom Force Field for Heterocycles. Properties of
Liquid Pyridine and Diazenes. J. Mol. Struct. 1998, 424, 145-155. DOI: 10.1016/S0166-1280(97)00237-6.

[41] Andersen, H. C. Molecular Dynamics at Costant Pressure And/or Temperature. J. Chem. Phys. 1980, 72, 2384-2393. DOI: 10.1063/1.439486.

[42] Zhao, Y.; De Nicola, A.; Kawakatsu, T.; Milano, G. Hybrid Particle-field Molecular Dynamics Simulations: Parallelization and Benchmarks. J. Comput. Chem. 2012, 33, 868-880. DOI: $10.1002 /$ jcc.22883.

[43] Berendsen, H. J. C.; van der Spoel, D.; van Drunen, R. GROMACS: A Message-passing Parallel Molecular Dynamics Implementation. Comput. Phys. Commun. 1995, 91, 43-56. DOI: 10.1016/00104655(95)00042-E.

[44] Berendsen, H. J. C.; Postma, J. P. M.; Van Gunsteren, W. F.; DiNola, A.; Haak, J. R. Molcular Dynamics with Coupling to an External Bath. J. Chem. Phys. 1984, 81, 3684. DOI: 10.1063/1.448118.

[45] Darden, T.; York, D.; Pedersen, L. Particle Mesh Ewald: An $\operatorname{Nlog}(\mathrm{N})$ Method for Ewald Sums in Large Systems. J. Chem. Phys. 1993, 98, 10089-10092. DOI: 10.1063/ 1.464397.

[46] Hess, B.; Bekker, H.; Berendsen, H. J. C.; Fraaije, J. G. E. M. LINCS: A Linear Constraint Solver for Molecular Simulations. J. Comput. Chem. 1997, 18, 1463-1472. DOI: 10.1002/(SICI)1096-987X (199709)18:12<1463::AID-JCC4>3.0.CO;2-H.

[47] Alfonso, G. C.; Yan, D.; Zhou, Z. Configurationalconformational Statistics of Atactic Polypropylene. Polymer (Guildf). 1993, 34, 2830-2835. DOI: 10.1016/ 0032-3861(93)90128-W.

[48] Allegra, G.; Bruckner, S. Copolymer Chain Statistics. The Pseudo-Stereochemical Equilibrium Approach within the Continuum of Rotational States. The Unperturbed Dimensions of Atactic Polypropylene. Macromolecules. 1977, 10, 106-113. DOI: 10.1021/ ma60055a022.

[49] Caputo, S.; De Nicola, A.; Donati, G.; David, A.; Raos, G.; Milano, G. All-Atom Model of Atactic 2-Vinyl Pyridine Polymer: Structural Properties Investigation by Molecular Dynamics Simulations. J. Electrochem. Soc. 2019, 166, B3309-B3315. DOI: 10.1149/2.0471909jes.

[50] Clancy, T. C.; Pütz, M.; Weinhold, J. D.; Curro, J. G.; Mattice, W. L. Mixing of Isotactic and Syndiotactic Polypropylenes in the Melt. Macromolecules. 2000, 33, 9452-9463. DOI: 10.1021/ma0011035.

[51] Daoulas, K. C.; Müller, M. Single Chain in Mean Field Simulations: Quasi-instantaneous Field Approximation and Quantitative Comparison with Monte Carlo Simulations. J. Chem. Phys. 2006, 125, 184904. DOI: 10.1063/1.2364506.

[52] Müller, M.; Daoulas, K. C. Calculating the Free Energy of Self-assembled Structures by Thermodynamic Integration. J. Chem. Phys. 2008, 128, 24903. DOI: 10.1063/1.2818565.

[53] Ladd, D. W. An Algorithm for the Binomial Distribution with Dependent Trials. J. Am. Stat. 
Assoc. 1975, 70, 333-340. DOI: $10.1080 /$ 01621459.1975 .10479867$.

[54] Larson, R. G. The Structure and Rheology of Complex Fluids; Oxford university press: New York, 1999; Vol. 150.

[55] Connection between Polymer Molecular Weight, Density, Chain Dimensions, and Melt Viscoelastic Properties. 1994.

[56] Liu, C.; Yu, J.; He, J.; Liu, W.; Sun, C.; Jing, Z. A Reexamination of GN0 and Me of Syndiotactic
Polypropylenes with Metallocene Catalysts. Macromolecules. 2004, 37, 9279-9282. DOI: 10.1021/ ma048743i.

[57] Fetters, L. J.; Lohse, D. J.; Graessley, W. W. Chain Dimensions and Entanglement Spacings in Dense Macromolecular Systems. J. Polym. Sci. Part B Polym. Phys. 1999, 37, 1023-1033. DOI: 10.1002/(ISSN)10990488. 